\title{
CHLORAMPHENICOL AND OTHER ANTIBIOTICS IN THE TREATMENT OF TYPHOID FEVER AND TYPHOID CARRIERS
}

\author{
By THEODORE E. WOODWARD, JOSEPH E. SMADEL, AND \\ HERBERT L. LEY, JR. \\ (From the University of Maryland School of Medicine, Baltimore, the Army Medical Depart- \\ ment Research and Graduate School, and the Commission on Immunization \\ of the Army Epidemiological Board, Washington, D. C.)
}

(Received for publication July 28, 1949)

The initial experience of our group with the beneficial effect of chloramphenicol in the treatment of ten Malayan patients with typhoid fever was published as a preliminary report (1). The work has been continued in the United States and we now wish to add to the earlier note detailed observations on 24 patients acutely ill with typhoid fever and five typhoid carriers who have received this antibiotic. In addition, the therapeutic results obtained in small groups of typhoid patients who were given aureomycin or polymyxin are included in this report.

\section{METHODS}

\section{Selection and observation of patients}

In all of the 33 treated acutely ill patients mentioned in the present study, the clinical diagnosis of typhoid fever was confirmed by the cultivation of Salmonella typhosa from their blood obtained prior to the initiation of specific therapy. Patients selected for treatment were in an active phase of the disease. In most instances therapy was begun within two weeks of onset of illness but two patients were first given chloramphenicol during a relapse of typhoid fever in the fifth and seventh weeks of illness. The course of the disease in the treated patients was followed by careful clinical observations and by laboratory examinations which included repeated cultures of samples of blood, feces and urine, as well as Widal tests, blood counts and other. procedures when indicated. In this study the patient's temperature was considered normal when the recording was below $99.0^{\circ} \mathrm{F}$. orally, or below $100.0^{\circ} \mathrm{F}$. rectally.

No attempt was made to treat alternate cases with chloramphenicol. During the period of the study, however, nine patients with typhoid fever were observed who received only supportive therapy. The diagnosis in each of these untreated patients was proved by the cultivation of $S$. typhosa from the blood or feces.

\section{Antibiotic therapy}

Chloramphenicol. Chloromycetin ${ }^{1}$ of the fermentation type was administered in the form of $0.25 \mathrm{gm}$. tablets or

\footnotetext{
1 Trade name, Parke, Davis and Company.
}

capsules which were furnished by Parke, Davis and Company. The bitter taste of the original experimental uncoated tablets was subsequently disguised by dispensing the drug in gelatin capsules. The usual therapeutic regime was as follows: Each patient received an initial large oral dose of 3.0 to $4.0 \mathrm{gm}$. of drug, calculated on the basis of approximately $50 \mathrm{mgm}$. per $\mathrm{kgm}$. of body weight. Throughout the febrile period the patients received a total of 2.0 to $3.0 \mathrm{gm}$. daily given in divided doses of $0.25 \mathrm{gm}$. every two or three hours. Therapy was continued for at least five days after the temperature returned to normal levels by administering $0.25 \mathrm{gm}$. tablets at four-hour intervals during the first few days and at six-hour intervals during the latter portion of this period. For various reasons, of which the most prominent was a shortage of chloramphenicol, a number of patients received smaller amounts of the antibiotic for a shorter time during the afebrile period than would have been given according to the schedule mentioned above. In the later period of the work a simplified regime was followed. Patients 20 through 23 received the usual large initial dose of drug after which they were given a total of $4.0 \mathrm{gm}$. daily in divided doses at eight-hour intervals during the febrile period; the dosage, still given at eight-hour intervals, was later reduced by a series of steps to $1.5 \mathrm{gm}$. daily. Vomiting was encountered rarely following the initial loading dose of drug; it occurred only when the bitter uncoated tablets were used and in no instance did it necessitate discontinuance of therapy.

In the majority of patients blood levels of chloramphenicol were determined at least once daily throughout the period of treatment. For this purpose serum was obtained from a specimen of blood taken immediately before the next dose of drug was due. Ley's modification ${ }^{2}$ of the turbidimetric microbiological method of Joslyn and Galbraith $(2,3)$ employing inhibition of growth of $S$. sonnei was used for the assay.

Aureomycin. Aureomycin, supplied in $0.25 \mathrm{gm}$. gelatin capsules by the Lederle Laboratories Division of the American Cyanamid Company, was administered orally to four American patients included in the present study. Two adults received an initial dose of $2.0 \mathrm{gm}$. followed by 0.50 to $0.75 \mathrm{gm}$. every four hours. Two children were given a smaller loading dose of $1.0 \mathrm{gm}$. and there-

2 This is available in detail in mimeographed form and will be furnished on request. 


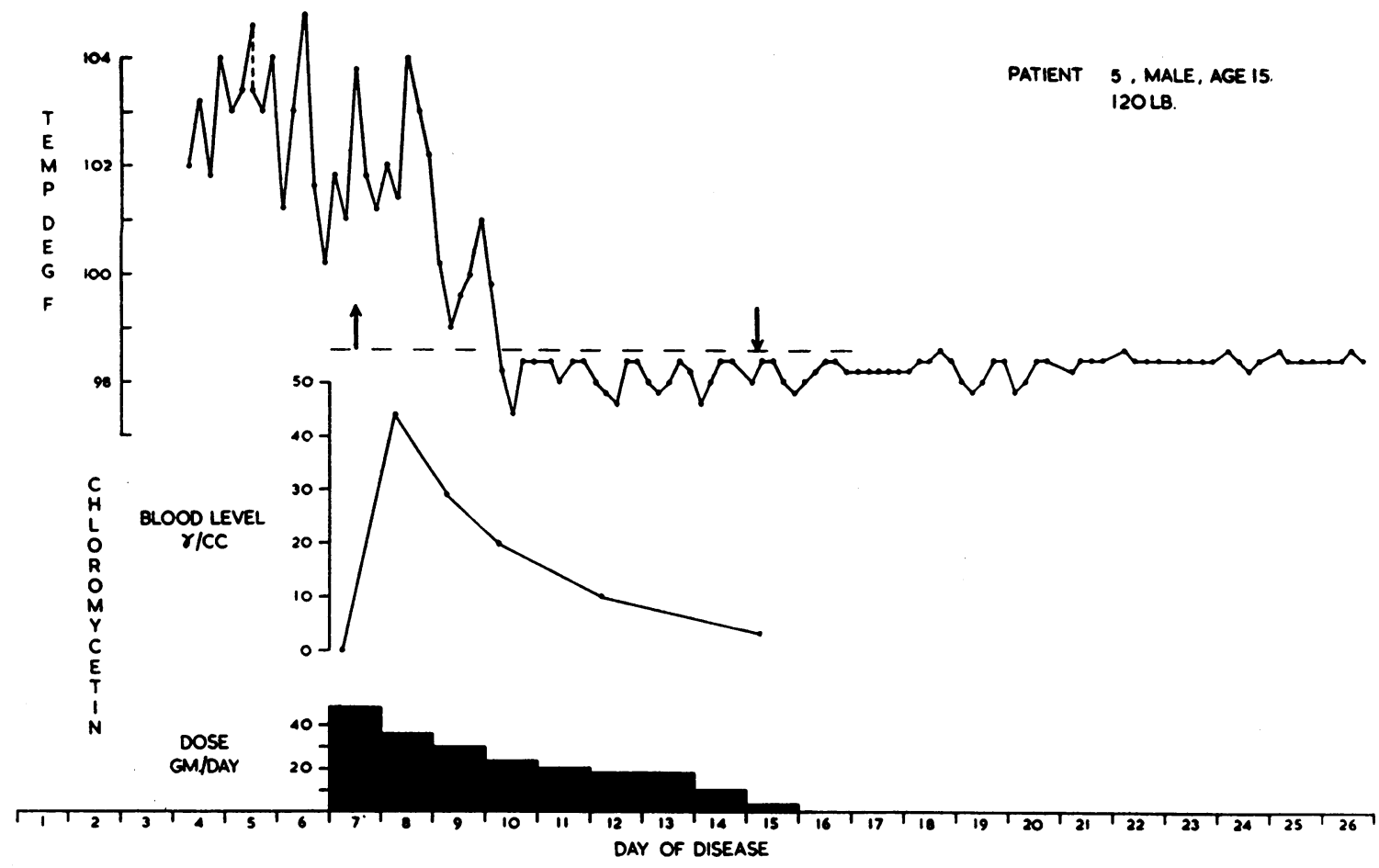

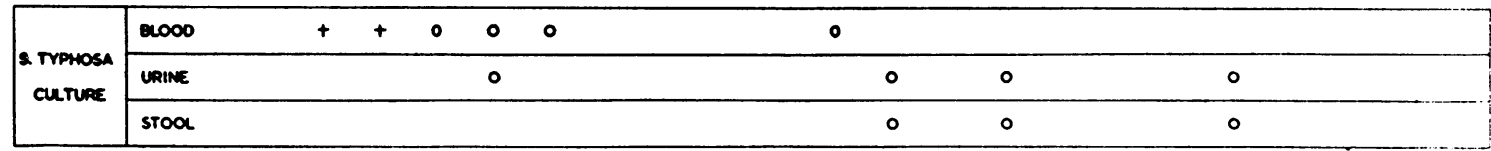

Fig. 1. Clinical Response of Typhoid Patient 5 to Chloramphenicol Therapy

A total of $21 \mathrm{gm}$. of chloramphenicol was given over a period of nine days.

after $0.25 \mathrm{gm}$. at four-hour intervals. Therapy was continued for two weeks in those patients who survived. Nausea and gastrointestinal irritation, such as has been noted by others (4), occurred in our patients but the regime was not interrupted because of this. Data on blood levels of aureomycin were not obtained.

Polymyxin. Vials of dried polymyxin hydrochloride and of special diluent were supplied by Lederle Laboratories. One cc. of a freshly prepared solution containing $40 \mathrm{mgm}$. of this antibiotic was administered intramuscularly every three hours for a period of about two weeks to five Chinese patients who were treated in Malaya. Certain toxic manifestations probably attributable to the polymyxin are discussed in the section on clinical results with this antibiotic.

In vitro tests for sensitivity of infecting organism to antibiotic agents

Infecting organisms from ten of the 11 Malayan patients who received chloramphenicol, and from all of those who were given polymyxin were tested for sensitivity to the antibiotic being used for treatment. A modification of the usual zone of inhibition technique was employed in which varying concentrations of antibiotic are placed in cups on a seeded agar surface. ${ }^{3}$ Organisms from the majority of the American patients who received chloramphenicol were tested for sensitivity by a turbidimetric technique using fluid cultures. Our observations with this method agree with those of Smith and his coworkers (3).

\section{RESULTS IN PATIENTS GIVEN CHLORAM PHENICOL}

\section{Observations in uncomplicated cases}

Some subjective or objective improvement was generally noted in patients acutely ill with typhoid fever within the first 36 hours after treatment was begun. However, by the third and fourth days of therapy, improvement was manifested by $(a)$ abatement of fever by lysis, $(b)$ disappearance of rose spots, which had been present in about half the patients, $(c)$ amelioration of headache, cough,

3 These tests were performed by Dr. Richard Green of the Institute for Medical Research, Kuala Lumpur. 
and the toxemic characteristics of this disease. Following the return of the temperature to normal levels on about the fourth day, convalescence generally proceeded at a rapid rate. The usual clinical response to chloramphenicol treatment is illustrated in the abbreviated case histories and graphical records of patients 5 and 7 which are presented below. The record of patient 1 (designated T-5 in the preliminary report) has already been published (1).

Patient 5. This 15-year-old Chinese boy entered the hospital on the fourth day of disease. His early illness was characterized by fever, headache, watery stools over a three-day period, and general malaise. The significant physical findings on admission were toxemia, dehydration, and a slightly tense and tender abdomen. Two pretreatment blood cultures were positive for $S$. typhosa. There was moderate albuminuria; the white blood cell count was 5,150, and the red blood cell count 4,400,000. Chloramphenicol treatment was initiated on the seventh febrile day and was continued for eight days. The course of fever, the daily amounts of drug given, the resultant blood levels, and the results of bacteriological examina- tions are represented in Figure 1. The patient was decidedly less toxic within 36 hours after initiation of treatment. Within 62 hours the temperature reached normal and the patient was clearly convalescent. Blood specimens taken on the first, second, third and eighth days of treatment were sterile. S. typhosa was not found in any stool or urine specimen examined. Convalescence was uneventful and rapid and the patient was discharged from the hospital on the 29th day after onset.

Patient 7. This 24-year-old Malay Indian was hospitalized on the sixth day of illness with the principal complaints of continued daily fever, anorexia, malaise, headache, and moderate cough. Examination revealed the usual signs of typhoidal toxemia including drawn facies, feeble dicrotic pulse and hypotension $(96 / 70)$; in addition, bilateral pulmonary rales and rhonchi, and a slightly distended and tender abdomen were noted. Two pre-treatment blood cultures contained $S$. typhosa. Specific treatment with chloramphenicol was instituted on the 12th febrile day. Rapid improvement of the general toxic state began about 36 hours later and the temperature remained permanently at normal levels after the fourth day of treatment. The antibiotic was administered for a total of eight days, i.e., three febrile and five afebrile days. White blood cell counts taken immediately

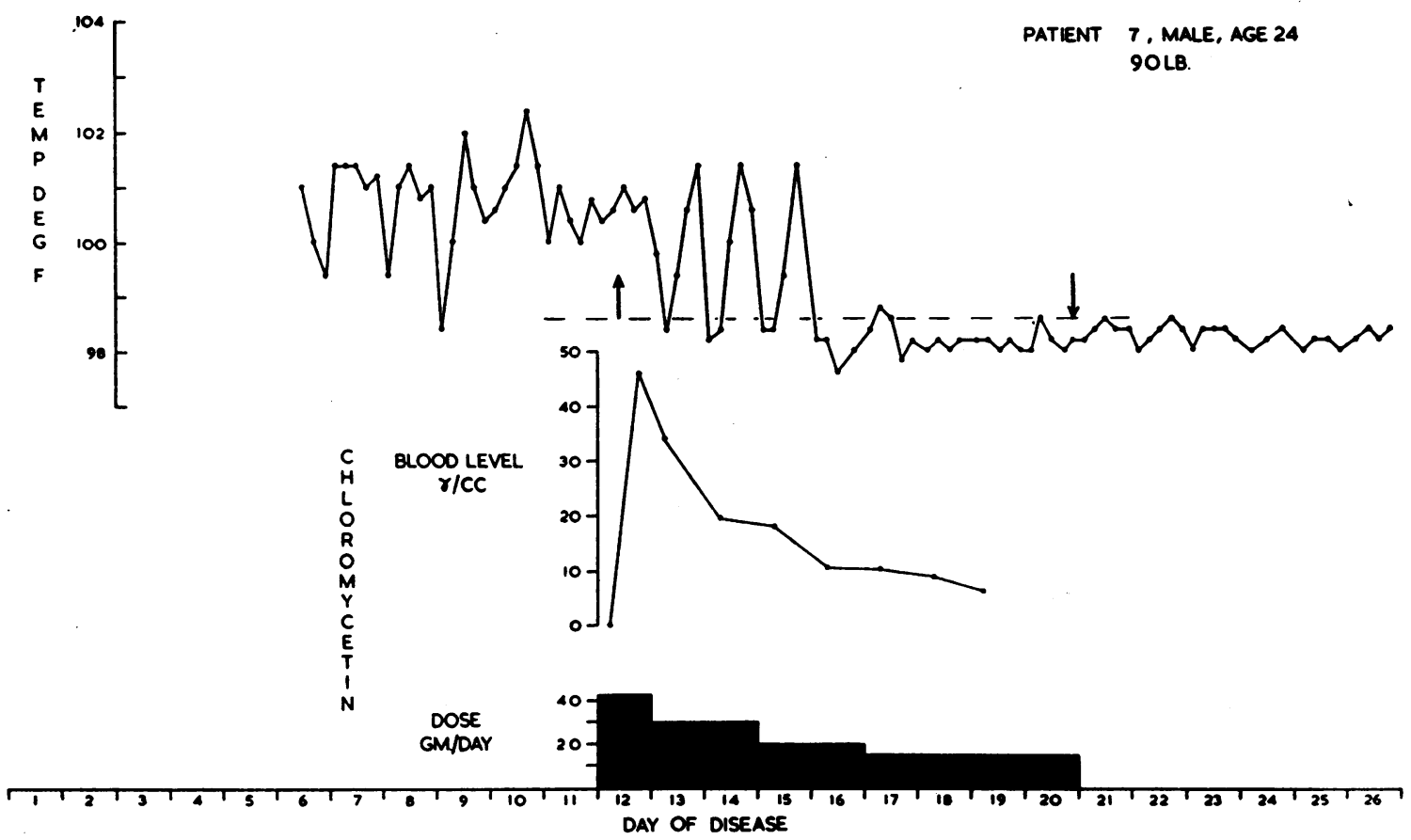

\begin{tabular}{|c|c|c|c|c|c|c|c|}
\hline \multirow{3}{*}{$\begin{array}{l}\text { rrpuces } \\
\text { curruas }\end{array}$} & 0000 & + & +0 & 0 & 0 & & \\
\hline & uance & & & 0 & 0 & 0 & 0 \\
\hline & stool & & & 0 & & 0 & 0 \\
\hline
\end{tabular}

Fig. 2. Clinical Response of Typhoid Patient 7 to Chloramphenicol Therapy A total of $20 \mathrm{gm}$. of chloramphenicol was given over a period of nine days. 


\begin{tabular}{|c|c|c|c|c|c|c|c|c|c|c|}
\hline & & & TYPF & HOID FI & EVER & $\begin{array}{l}\text { TABLE } \\
\text { TREATED }\end{array}$ & $\begin{array}{l}\text { EI } \\
\text { WTTH CH }\end{array}$ & LORAMPH & ENICOL & . \\
\hline \multicolumn{3}{|c|}{ PATIENT * } & \multicolumn{3}{|c|}{ TREATMENT } & \multicolumn{3}{|c|}{ RESPONSE AFTER TREATMENT } & \multirow{3}{*}{$\begin{array}{l}\text { LAST } \\
\text { FEBRILE } \\
\text { DAY OF } \\
\text { DISEASE }\end{array}$} & \multirow{3}{*}{$\begin{array}{l}\text { LAST DAY OF } \\
\text { OISEASE } \\
\text { S. TYPHOSA } \\
\text { FOND IN FECES }\end{array}$} \\
\hline \multirow{2}{*}{ No. } & \multirow{2}{*}{$A G E$} & \multirow{2}{*}{ SEX } & \multirow{2}{*}{$\begin{array}{l}\text { DAY of } \\
\text { DASENSE } \\
\text { BEGUN }\end{array}$} & \multirow{2}{*}{$\begin{array}{l}\text { DAYS } \\
\text { CONTINUED }\end{array}$} & \multirow{2}{*}{$\begin{array}{l}\text { TOTAL } \\
\text { DRUG } \\
\text { (GM.) }\end{array}$} & \multirow{2}{*}{$\begin{array}{c}\text { DURATION OF FEVER } \\
\text { AFTER TREATMENT } \\
\text { (OAYS) }\end{array}$} & \multicolumn{2}{|c|}{ CONPLICATIONS } & & \\
\hline & & & & & & & NATURE & $\begin{array}{l}\text { ONSET, DAY } \\
\text { OF OISEASE }\end{array}$ & & \\
\hline 1 & 24 & $\mathbf{F}$ & 9 & 9 & 22 & 2 & NONE & & 11 & 21 \\
\hline 2 & 25 & M & 9 & 10 & 25 & 4 & NIESTNAA & 16 & 12 & 0 \\
\hline 3 & 32 & $M$ & 17 & 9 & 28 & 3 & PERFORATION & 21 & 28 & 18 \\
\hline 4 & 9 & $\mathbf{F}$ & 10 & 8 & 15 & 3 & NONE & & 13 & 0 \\
\hline 5 & 15 & M & 7 & 9 & 21 & 3 & NONE & & 10 & 0 \\
\hline 6 & २5 & $\mathbf{F}$ & 12 & 8 & 17 & 3 & RELAPSE & 31 & 15 & 28 \\
\hline 7 & 24 & $M$ & 12 & 9 & 20 & 4 & NONE & & 16 & 0 \\
\hline 8 & 30 & M & 8 & 5 & 12 & 4 & RELAPSE & 23 & 12 & 50 \\
\hline 9 & 20 & M & 7 & 8 & 20 & 3 & NONE & & 10 & 0 \\
\hline 10 & 35 & $\mathbf{F}$ & 10 & 9 & 25 & 4 & NONE & & 14 & 0 \\
\hline "I & 28 & $\mathbf{F}$ & 9 & 7 & 17 & 5 & RELAPSE & 32 & 14 & 33 \\
\hline 12 & 4 & $\mathbf{F}$ & 12 & 4 & 8 & 2 & NONE & & 14 & 0 \\
\hline 13 & 8 & M & 20 & 11 & 22 & 4 & NONE & & 24 & 23 \\
\hline .14 & 9 & $\mathbf{F}$ & 12 & 10 & 20 & 3 & NONE & & 15 & 14 \\
\hline 15 & 74 & $M$ & 7 & 16 & 54 & 4 & $\begin{array}{l}\text { NTESTNAN } \\
\text { HEMORAHAGE }\end{array}$ & 9 & 26 & 13 \\
\hline 16 & 37 & $\mathbf{F}$ & 13 & 7 & 20 & 4 & RELAPSE & 31 & 17 & 38 \\
\hline 17 & 5 & $\mathbf{F}$ & 10 & 14 & 25 & 3 & NONE & & 13 & 18 \\
\hline B & 39 & $M$ & 16 & 12 & 46 & 5 & NONE & & 21 & 15 \\
\hline$\theta$ & 10 & $\mathbf{F}$ & 14 & 12 & 25 & 3 & NONE & & 17 & 15 \\
\hline 20 & 33 & $\mathbf{F}$ & 16 & 12 & 32 & 4 & NONE & & 20 & 22 \\
\hline 21 & 29 & $\mathbf{F}$ & 21 & 6 & 25 & 3 & NONE & & 23 & 0 \\
\hline 22 & 6 & $F$ & 11 & 8 & 16 & 4 & NONE & & 15 & 0 \\
\hline AV. & & & 11.9 & 9.2 & 23.4 & 3.5 & & & & \\
\hline 231 & 7 & F & 35 & 12 & 20 & 2 & NONE & & 37 & 33 \\
\hline $24 !$ & B & $M$ & 46 & 7 & 17 & 3 & NONE & & 49 & 49 \\
\hline - s.t & IPPAOS & ISA WATE: & AS CULTI & $\begin{array}{l}\text { JRED FROM } \\
\text { STOOL SPEC }\end{array}$ & $\begin{array}{l}\text { QLOOD } \\
\text { AMENS }\end{array}$ & $\begin{array}{l}\text { TANEN PAOOR TO TI } \\
\text { WERE REPEATEOUY } \\
\text { IRAMG A RELAPSE }\end{array}$ & EATMENT FRO & $\begin{array}{l}\text { M EACH PATIE! } \\
\text { S. TYPHOSA }\end{array}$ & Uat & $\operatorname{lon}$ \\
\hline
\end{tabular}

before treatment and in early convalescence were 7,200 and 7,100 , respectively. Numerous cultures of blood, urine and stool specimens taken after therapy was begun were negative for typhoid bacilli. Convalescence was rapid and the patient was discharged on the 30th day of disease. Figure 2 represents the graphic chart of the clinical record of this patient.

A summary of the results obtained in the 24 cases of typhoid fever who received chloramphenicol is presented in Table I. Eight of the patients were children; the majority of the adults were in the third and fourth decades but one was 74 years of age. There were a few more females than males in the group. Drug therapy was begun on the seventh to the 21 st day, average 11.9 , of the initial attack of the 22 patients. Irrespective of the duration or severity of the disease, or of the age of the patient, the temperature returned to normal levels within five days after chloramphenicol therapy was instituted. The average duration of fever after beginning treatment was approximately 3.5 days in these 22 patients. Two persons who were first treated during a relapse received the drug on the 35th and 46th days after onset of their disease ; these were the eighth and fifth febrile days of the relapses. Both responded promptly, one becoming permanently afebrile in two days and the other in three.

The majority of the patients made an uneventful recovery, but the course was complicated in seven of the 22 who were treated during their initial febrile episode. 


\section{Observations in cases with complications}

Intestinal hemorrhage and perforation. Two of the usual complications of typhoid fever, namely, intestinal hemorrhage and intestinal perforation, developed in three patients in the treated series. Severe intestinal hemorrhage occurred in patient 2 and.moderate bleeding in patient 15 ; both made satisfactory recoveries following supportive treatment which included blood transfusions. The first patient began to bleed on the 16th day of the disease, or the seventh day of therapy, at which time his temperature was normal. The second showed signs of bleeding while still febrile on the ninth day of the disease or the second day of treatment. A brief abstract of the findings in patient 3 , who developed intestinal perforation, is given below, and a graphic chart of his clinical record is reproduced as Figure 3.
Patient 3. This severely ill 32-year-old Chinese male entered the hospital on the 12th day of an illness characterized by continuous fever, headache, abdominal pain and distention, occasional vomiting, and an irritating, nonproductive cough. On the 17 th day of illness, when treatment was begun, additional findings consisted of muttering delirium, pulmonary rales, markedly increased abdominal distention and numerous rose spots. The blood pressure was $110 / 70$; the pulse, 124 ; the white blood cell count, 4,600; the red blood cell count, 1,140,000; and the urine contained moderate amounts of albumin. Blood samples taken on the 15th day of illness and on the 17th day, immediately prior to the initial dose of drug, yielded $S$. typhosa upon culture.

The patient's response to chloramphenicol treatment was satisfactory as shown by the reduced toxemia, the gradual clearing of the mental state, the return of appetite and the lessening of abdominal tenderness and distention. On the third day of therapy the temperature reached normal levels and remained there for the next 36 hours. The lungs were then clear, the rose spots had disappeared and the pulse and heart sounds were of better

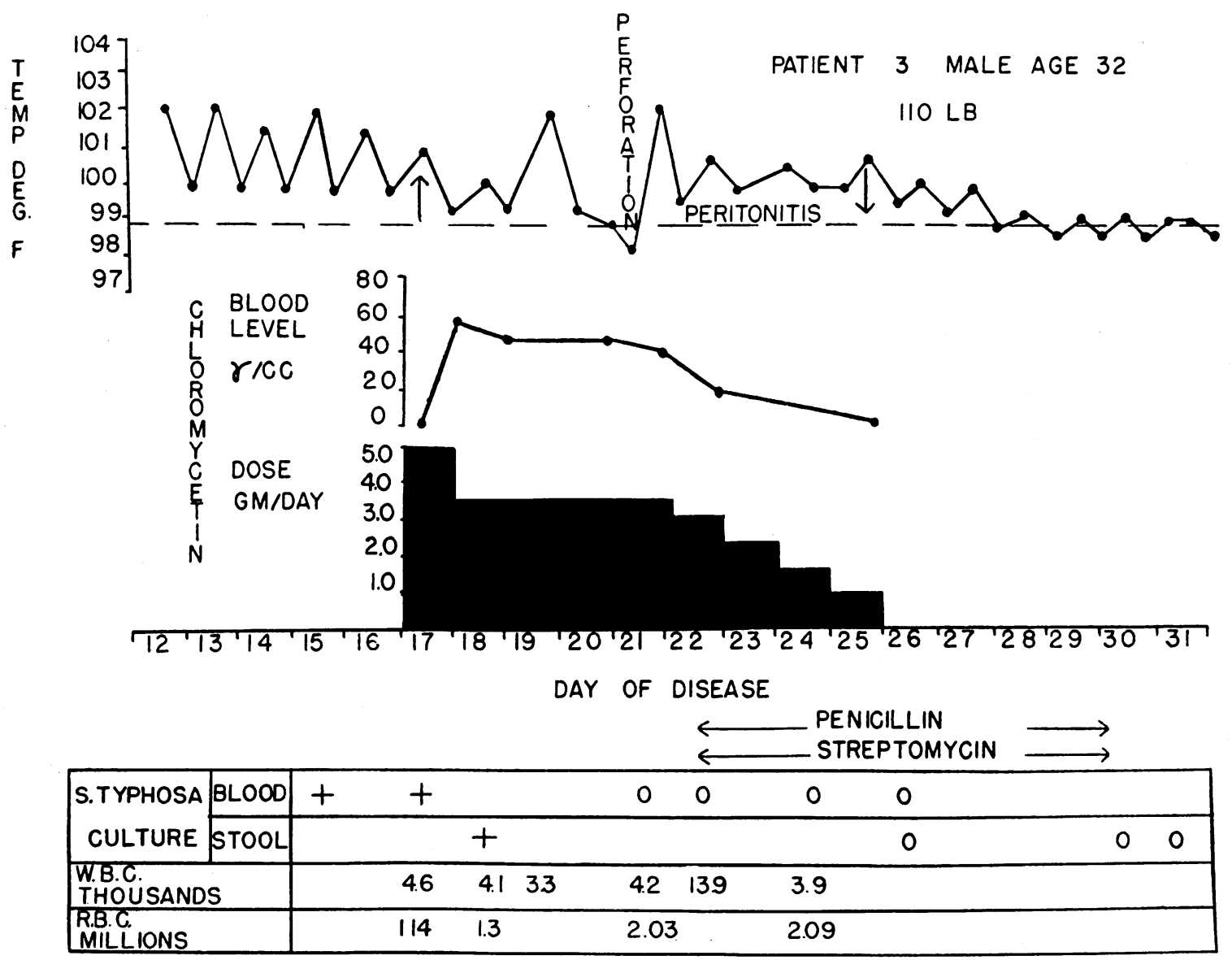

Fig. 3. Clinical Response of Typhoid Patient 3 to Chloramphenicol Therapy

Early convalescence was complicated by intestinal perforation on the 21 st day of disease. The patient received a total of $28 \mathrm{gm}$. of chloramphenicol over a period of nine days. 
quality. The patient, now rational, complained of constipation and slight abdominal distention. At this point, on the 21st day of illness, an unwise medical attendant gave, without orders, $1.0 \mathrm{cc}$. of pituitrin in an effort to relieve the gaseous distention. Apparently the increased peristalsis accompanied by a local rise of intra-intestinal pressure, which followed the pituitrin injection, resulted in a perforation presumably at the site of a typhoid ulcer. When observed several hours after this episode, the patient was in a state of collapse. Intravenous fluids and blood transfusions were given as supportive measures, and in addition, chloramphenicol therapy was supplemented by the administration of large doses of penicillin and streptomycin. During the next 12 hours the condition of the patient remained critical. There was evidence of pneumoperitoneum and severe generalized peritonitis, and the white blood cell count rose to 13,900 . Some improvement was observed after 18 hours, and at the end of 36 hours it appeared that the patient would survive. By the third day after perforation active peristalsis was audible and the patient passed soft fecal material in the usual manner. From this point onward the improvement was steady; the temperature dropped to normal levels by the sixth day after perforation. About the time that the patient became afebrile the abdomen was sufficiently soft to allow palpation of a mass in the right lower quadrant; this was discernible for a week before it finally disappeared.

$S$. typhosa was cultured from the stool sample obtained on the 18th day of illness, i.e., 24 hours after therapy was begun. Typhoid organisms were not grown from subsequent specimens of stool or blood. The patient was discharged from the hospital on the 46th day after onset of disease.

At the time of intestinal perforation surgical intervention was intensively considered but the opinion prevailed that the patient was in too poor a condition to survive any surgical procedure. Therefore, it was decided to attempt to control the infection with the aid of combined antibiotic therapy until the perforation could be sealed off by natural processes. The results were so satisfactory that the idea of surgical intervention was no longer considered after the patient became a suitable operative risk.

Relapses of typhoid fever. Four of the 22 patients treated during their initial illnesses developed relapses. These persons (patients $6,8,11$, and 16) had been started on chloramphenicol therapy between the eighth and 13th days of their disease and had responded in the usual manner. The relapses occurred between the 23rd and 32nd days after the onset of typhoid fever, which represented periods of ten to 16 days after drug had been discontinued or 11 to 18 days after the patients had become afebrile.

The recrudescent disease in each of these individuals was characterized by fever, mild toxemia, and the presence of $S$. typhosa in the blood and feces. One of the patients, No. 11, was not given specific therapy during her relapse because of an inadequate supply of the antibiotic. She ran a moderately severe course with fever for ten days. The remaining members of the group were treated between the first and fourth days of their relapse. The duration of the recurrent fever after treatment was begun varied between one and one-half and three days. S. typhosa disappeared promptly from the blood and stools of the three treated patients and did not reappear. An abstract of the case history of one of the patients whose disease relapsed and a graphic summary of the data represented in Figure 4 are presented below.

Patient 6. A 25-year-old Chinese girl was hospitalized on the ninth day of a febrile illness characterized by continuous fever, headache, moderate abdominal pain and delirium. She showed toxemia and dehydration and there were bilateral pulmonary rales; both the liver and spleen were palpable. Two blood cultures on the 11th and 12th days of illness contained $S$. typhosa. The white blood cell count on admission was 2,700 and the red blood cell count was $3,720,000$. On the 12th day of illness an initial oral dose of $2.2 \mathrm{gm}$. of chloramphenicol was administered. The drug was continued with $0.2 \mathrm{gm}$. doses every two hours for the succeeding eight days, which was five days after the temperature became normal. A total of $16.5 \mathrm{gm}$. of drug were given.

Improvement in the clinical condition was noted after two days of antibiotic treatment and the temperature reached normal levels in less than three days. Specimens of blood taken during the second, third, and fourth days of treatment were sterile, and frequent samples of stool and urine obtained during the third week of illness were free of bacilli.

On the 31st day of illness, 16 days after the patient became afebrile and 12 days after the last dose of chloramphenicol, the patient complained of headache and that afternoon the temperature reached $102.2^{\circ} \mathrm{F}$. The blood was again found to contain S. typhosa. On the second day of this clinical relapse chloramphenicol treatment was reinstituted; an initial dose of $3.0 \mathrm{gm}$. was followed by doses of $0.25 \mathrm{gm}$. every two hours for seven days. Abatement of the headache was rapid and the temperature reached normal in one and one-half days after the second course of therapy was started. The convalescence thereafter was uneventful and later blood and stool cultures did not yield typhoid organisms.

The prompt clinical response of these individuals indicated that the infecting organism responsible for the relapse was not appreciably resistant to chloramphenicol. This was further established by in vitro laboratory tests which showed that the 
sensitivity of the organism isolated from three of the patients during their relapses was essentially the same as the sensitivity of the organisms isolated prior to treatment.

It is to be noted that in each of the four patients with relapses the initial course of chloramphenicol had been given for a period of eight days or less. Indeed, the patients had been afebrile for from one to five days, average three, when drug was stopped. The relatively high incidence of relapses, two in ten, in the early portion of the present studies led us to the opinion that chloramphenicol therapy should be continued for a longer period of time after the patients became afebrile. Subsequent observations during the latter portion of the present work and in studies by another Army group in Malaya (5) appeared to confirm our impression. In fact, analyses of 44 cases treated by the two groups showed that about half the patients who received chloramphenicol for eight days or less suffered relapses of typhoid fever, whereas none of those treated for 12 days or longer did so.

\section{Laboratory findings in patients treated with} chloramphenicol

It was the general experience that within two hours after therapy was begun the patient's blood no longer contained demonstrable $S$. typhosa.
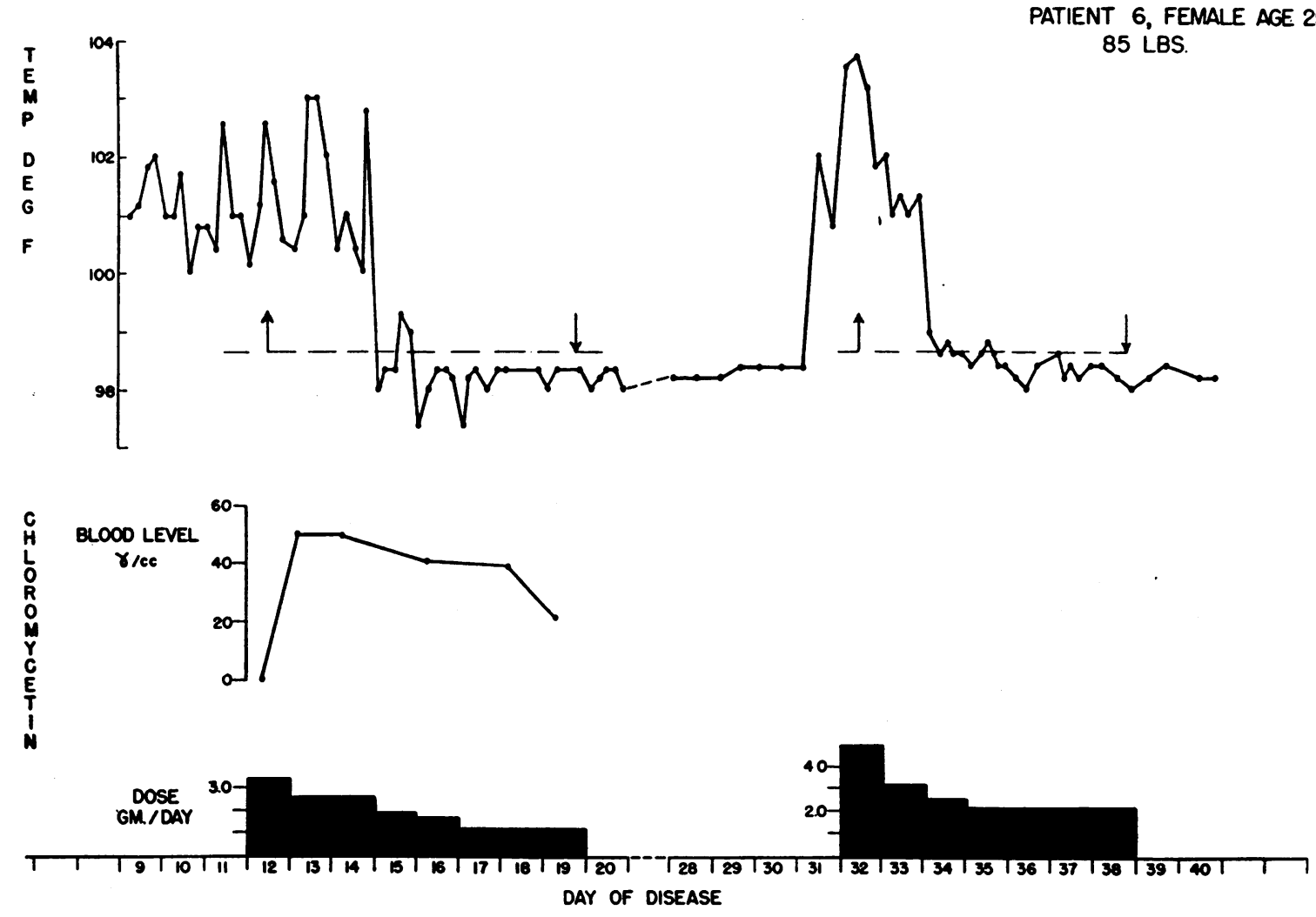

\begin{tabular}{|c|c|c|c|c|c|c|c|c|c|c|c|c|}
\hline \multirow{3}{*}{$\begin{array}{l}\text { STPPHOSA } \\
\text { QIIUURE }\end{array}$} & BLOOD & + & + & 0 & 0 & 0 & & & + & 0 & & \\
\hline & URINE & & & & 0 & & 0 & 0 & 0 & 0 & 0 & 0 \\
\hline & STOOL & & & & 0 & 0 & 0 & + & 0 & 0 & 0 & 0 \\
\hline
\end{tabular}

Fig. 4. Clinical Response of Typhord Patient 6 to Chloramphenicol Therapy

A total of $17 \mathrm{gm}$. of chloramphenicol was given over a period of eight days during the initial illness. A clinical relapse occurred 12 days after treatment was discontinued, but this responded promptly to a second course of drug. 
Early in the work frequent cultures taken at intervals of several hours throughout the first day of treatment consistently gave negative results. There was an inclination to discount these observations since no method was available for inactivating chloramphenicol, and it was thought possible that sufficient drug might be present in the sample to prevent growth. Subsequent observations on four of the patients in this series indicated that such might not be the principal determining factor $^{4}$ in the negative results. $S$. typhosa were grown from specimens taken from two patients 24 hours after therapy was initiated and from specimens obtained from two other patients at 48 hours. Except for these positive results and the cultures obtained during the clinical relapses, bacteremia was not demonstrated after therapy was begun.

Nine of the 24 patients listed in Table I did not have demonstrable $S$. typhosa in their stools at any time during the course of observation. Ten of the 24 patients had $S$. typhosa in their stools prior to treatment but two of these (patients 18 and 23) did not excrete these organisms at any time after drug was administered. Among the remaining $13, S$. typhosa occurred on one or more occasions after beginning chloramphenicol therapy; five of these had positive cultures after the drug was discontinued and, of these, four developed typical clinical relapses. None of the patients in the present group developed the chronic carrier state during the period of observation. Each individual provided three consecutive stool specimens which were negative for $S$. typhosa before being discharged from the hospital. In Table $I$ is given the last day of the disease on which $S$. typhosa was found in the stool of each patient. No cultures of urine samples from the patients in the present series yielded $S$. typhosa either before or after treatment.

The maximum titers attained in the Widal tests performed with type "O" antigen in all 24 patients ranged from $1: 40$ to $1: 2560$, geometric mean,

\footnotetext{
4 The routine method for performing blood cultures was as follows: 3-5 cc. of fresh venous blood were immediately inoculated into approximately $10 \mathrm{cc}$. of fluid media. After an interval of one to 18 hours of incubation at $37^{\circ} \mathrm{C}$., streak subcultures of the mixture of blood and broth were made on E. M. B. agar. Following appropriate incubation, these plates were examined in the usual manner and pathogenic members of the enteric group were identified by agglutination with specific antisera.
}

$1: 470$. Relapses of typhoid fever were not correlated with titers of " $O$ " agglutinins. At the time of the relapses values of $1: 40,1: 640,1: 880$, and $1: 1000$ were recorded for the four persons who suffered from exacerbations of the disease.

Blood levels of chloramphenicol were determined throughout the course of treatment in 19 of the patients. The data presented in Figures 1 to 4 illustrate the typical findings. During the first day the levels usually ranged between 40 and 80 gamma /cc. but on occasion values as high as 100 were attained. During the subsequent three to five days the levels generally ranged between 20 and 40 gamma/cc. Those patients treated late in the series with a prolonged course of drug received $1.5 \mathrm{gm}$. daily in divided doses of $0.5 \mathrm{gm}$. at intervals of eight hours during the last five to seven days of treatment. While frequent blood levels were not obtained in this group, other observations (6) indicate that oral doses of $0.5 \mathrm{gm}$. of chloramphenicol provide blood levels of 10 to 20 gamma/cc. during the first few hours after the dose is given and that the level is barely detectable at the end of eight hours.

In this group of treated patients, as in others previously studied $(1,7-9)$, there were no evidences of toxic manifestations attributable to the chloramphenicol. Abnormalities in the white and red blood cells and in the urine were recorded in a number of instances but were considered to be part of the disease picture of typhoid or of the complications which developed.

\section{The effect of chloramphenicol on the typhoid car- rier state}

Results of therapy. Four adult women, who were known to have been carriers of $S$. typhosa for ten or more years, were studied bacteriologically until two or more specimens of duodenal contents or stool were shown to contain typhoid bacilli. Following proof of the carrier state, each patient received an initial oral dose of $60 \mathrm{mgm}$. per $\mathrm{kgm}$. of body weight of chloramphenicol; this was followed by a total of 4.0 to $6.0 \mathrm{gm}$. daily given in divided doses every six hours. Each patient received an average of $71 \mathrm{gm}$. of the antibiotic over a 15-day period. High blood concentrations were obtained without exception; the over-all average was $38 \mathrm{gamma} / \mathrm{cc}$. An attempt was made to follow the antibiotic level in bile ob- 
tained by duodenal aspiration two hours after each dose was given, but results were erratic and unreliable, presumably because of unabsorbed drug present in the duodenal fluids.

Duodenal drainage fluid from each patient became negative for $S$. typhosa within two to six days after therapy was initiated, and remained so for the duration of treatment. However, in the majority of instances, the concentrations of chloramphenicol in these samples were sufficiently high to be expected to inhibit growth of the organisms in the culture media. In any case the apparent bacteriologic sterilization of the duodenal contents was probably of little significance since $S$. typhosa reappeared in all four patients shortly after cessation of treatment. Cultures of the stool, with few exceptions, were uniformly positive for $S$. typhosa throughout the period of treatment. No adequate explanation is at hand for these continued positive stool cultures during the course of intensive therapy.

The removal of the gallbladder from one patient

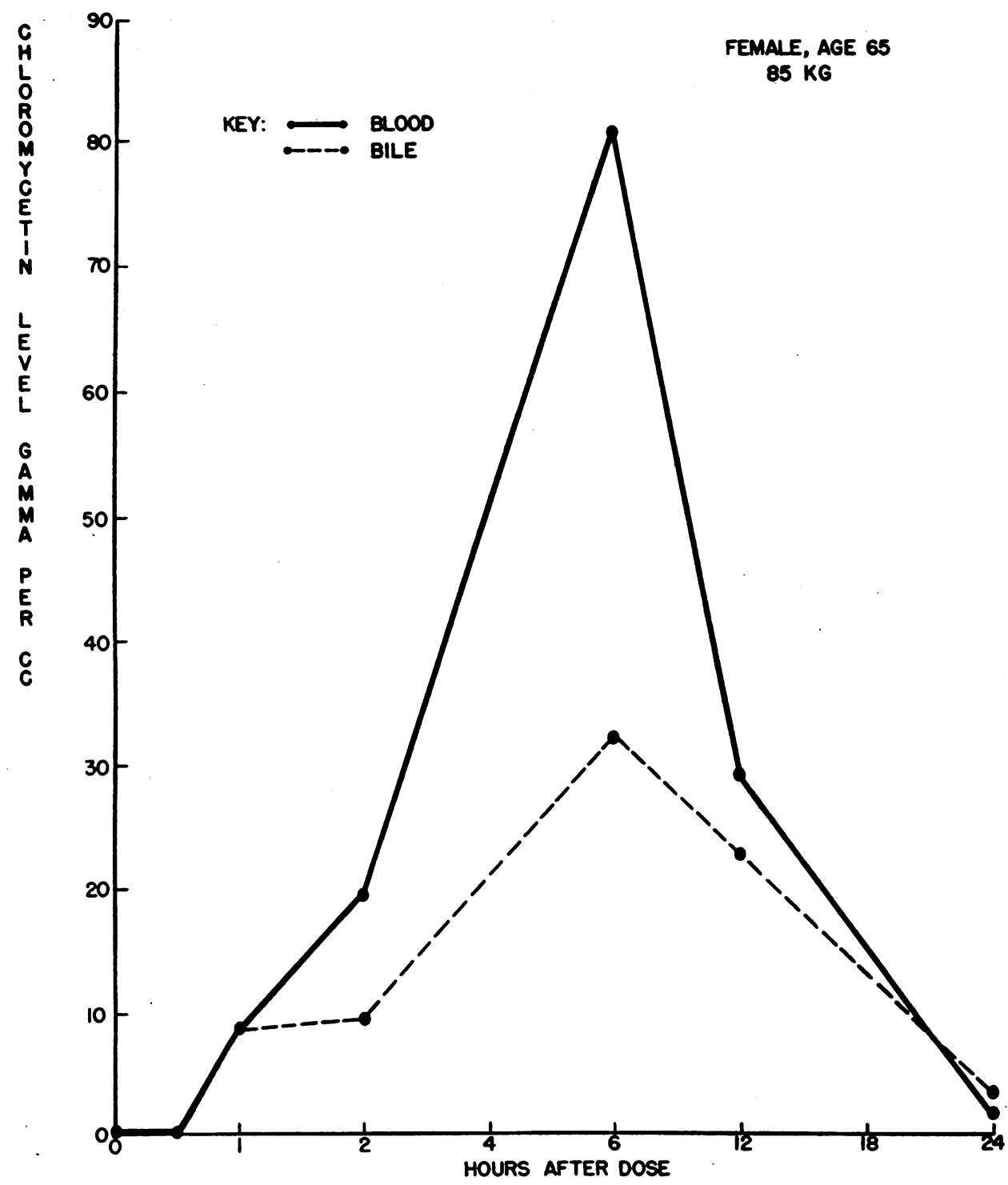

Fig. 5. Simultaneous Chloramphenicol Levels in Blood and Bile After 4.0 Gm. Oral Dose

See text for details and interpretation. 
(patient D) during the period of treatment allowed a careful bacteriologic study to be done. Cultures of bile, obtained from the common duct and from the gallbladder, and also from scrapings of the wall of the bladder itself, gave negative results for $S$. typhosa. However, a crushed stone yielded a positive culture for typhoid bacilli. It is of interest that $S$. typhosa subsequently reappeared in the duodenal contents of this patient in spite of cholecystectomy and chloramphenicol therapy.

These observations indicate that chloramphenicol therapy in four chronic typhoid carriers was consistently unsatisfactory in eradicating the carrier state.

Chloramphenicol levels in bile of human beings. Two patients suitable for the study of biliary excretion of chloramphenicol were observed during the period covered by this report. The first of these, an adult female, had a carcinoma of the stomach without evidence of biliary obstruction. She was given a single oral $4.0 \mathrm{gm}$. dose of chloramphenicol three hours prior to an exploratory laparotomy. At the time of the operation simultaneous specimens of blood and bile were obtained, the latter by direct aspiration of the gallbladder. Bioassay $^{5}$ of these samples revealed antibiotic levels of 69 and 27 gamma/cc. in blood and bile, respectively.

The second was the chronic typhoid carrier (patient D) whose gallbladder had been removed recently. She was studied during convalescence from the operation at the time that a "T tube" drain was present in the common duct. On two occasions single oral doses of 2.0 and $4.0 \mathrm{gm}$. of chloramphenicol were given to this patient. Bioassay of samples of blood and bile taken simultaneously at intervals after the oral dose demonstrated that antibiotic levels in the bile were approximately 50 per cent of those in the blood, although the maximum levels reached were dependent, of course, on dosage. The results obtained in this patient with the $4.0 \mathrm{gm}$. test dose are shown in Figure 5.

\footnotetext{
5 The bioassay technique of Joslyn and Galbraith $(2,3)$ must be modified to allow satisfactory estimation of chloramphenicol levels in bile. The specimen is centrifuged to remove all particulate matter and the clear supernatant fluid is used in the assay as though it were serum. To reduce non-specific light absorption due to the color of the bile, the optical densities of the tubes of the assay are read at a wave-length of 800 millimicrons.
}

RESULTS IN PATIENTS GIVEN AUREOMYCIN AND POLYMYXIN

\section{Aureomycin}

Four American patients with typhoid fever were given aureomycin orally beginning on the 12 th to 16 th day after onset of illness. Two of the patients died. One, a seven-year-old boy, succumbed to the toxemia of the disease on the 22nd day of illness after receiving a total of $13.8 \mathrm{gm}$. of drug over seven days. His sister, patient 12 in Table I, recovered promptly on chloramphenicol therapy. The other fatal case was a 42-year-old man who died on the 19th day of disease after taking $13.0 \mathrm{gm}$. of antibiotic during the three days prior to death. In the two remaining patients some reduction in fever occurred for a few days after instituting therapy but this was not accompanied by an obvious decrease in the toxemic manifestations of typhoid fever. Figure 6 illustrates graphically the course of disease in one of these patients who received a total of $59.1 \mathrm{gm}$. of aureomycin between the 12th and 26th days of illness and became afebrile after the period covered by the chart. The other patient who survived was given $22.2 \mathrm{gm}$. of drug between the 15th and 28th days and remained afebrile after the 27 th day of disease.

Although our experience with aureomycin in typhoid fever has been limited, it has been uniformly discouraging. This is in contrast to the equivocal preliminary results reported by Collins and his coworkers (4).

\section{Polymyxin}

Five Chinese patients in Malaya who were severely ill with typhoid fever were treated with polymyxin. The mean day of illness on which therapy was begun was 17 , range 10 to 27 ; the total course of drug administered per patient averaged $3.1 \mathrm{gm}$. given over 13.8 days; the duration of fever after treatment was instituted averaged 22 days, range 17 to 27 ; and the last febrile day of primary illness, exclusive of relapses, averaged 38.6 days. There were no fatalities. One patient suffered a moderately severe intestinal hemorrhage and another had severe furunculosis which responded to treatment with penicillin. Three of the five members of this group suffered relapses of typhoid fever during which $S$. typhosa was again recovered from their blood or stool. Two of the 


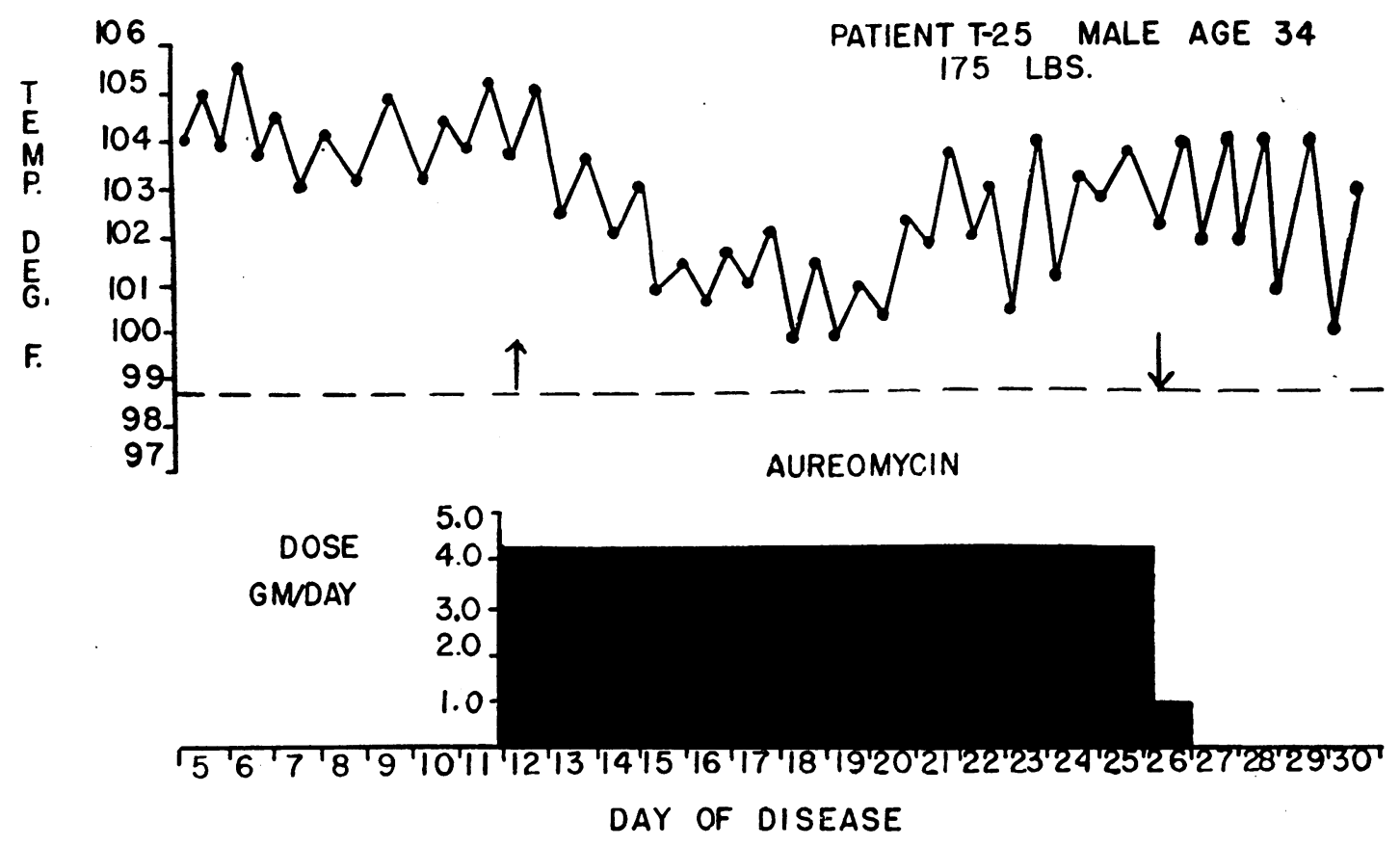

\begin{tabular}{|c|c|c|c|c|c|c|c|}
\hline WB.C. & & 6,300 & 3050 & & & & \\
\hline S. TYPHOSA & BLOOD & ++ & $++t$ & $+t$ & & & 0 \\
\hline CULTURE & STOO & & & 0 & 0 & 0 & 0 \\
\hline
\end{tabular}

Fig. 6. Unsatisfactory Clinical Response of Typhoid Patient T-25 to Aureomycin Therapy A total of $59 \mathrm{gm}$. of aureomycin was given over a period of 14 days.

patients whose blood was cultured at frequent intervals after treatment was begun continued to show bacteremia for three and six days, respectively, during therapy. Two of the patients developed severe albuminuria accompanied by numerous erythrocytes and casts in the urinary sediment; renal damage has been noted by others (10) following polymyxin therapy. Polymyxin apparently elicited no beneficial effects in these patients; indeed they pursued an unaltered course of severe typhoid fever. Although unsatisfactory clinical results were obtained, the strains of $S$. typhosa recovered from each of the patients displayed a high degree of sensitivity to polymyxin when tested in vitro; concentrations of the order of $5 \mathrm{gamma} / \mathrm{cc}$. produced a zone of inhibition of approximately $23 \mathrm{~mm}$. when the organisms were examined by the cup-agar plate method.

The in vitro sensitivity of the Malayan strains of $S$. typhosa to polymyxin, although tested by a different method, was of about the same order of magnitude as that observed by others in the United States (11).

Our clinical observations with polymyxin in typhoid fever were in contrast to the somewhat encouraging results reported by Schoenbach and his associates (10).

\section{COURSE OF TYPHOID FEVER IN PATIENTS RECEIVING} NO ANTIBIOTIC THERAPY

During the present studies nine patients with typhoid fever were observed who did not receive antibiotic therapy in any form. Eight of these were seen in Kuala Lumpur and one in Baltimore. In this group two deaths occurred, one on the 17 th and the other on the 33rd day after onset of illness. Both of these displayed extreme toxemia and died as a result of the severity of the disease without recognizable complications. The average duration of fever in the remaining seven cases was 
35 days. The course in these seven was not complicated by hemorrhage or perforation.

The observations on these untreated individuals agree in general with those given in one of the last published reports on typhoid fever from the Institute for Medical Research in Kuala Lumpur prior to the Japanese occupation. The following paragraph summarizes the results in an explosive outbreak presumably traced to a single carrier.

"Fifty-three cases were closely observed in hospital and a complete clinical summary was made. There were great variations in clinical response to what was presumably the same strain, or related strains, of the infecting bacillus. Such variations ranged from mild symptoms among ambulant cases to severe toxaemia and death. Thirteen per cent died either from toxaemia or intestinal haemorrhage; 8 per cent relapsed but recovered; and there were a number of disabling complications and sequelae. Among all cases, 8 per cent had peripheral neuritis (one man, severely affected, was disabled for more than five months); and 4 per cent developed typhoid abscesses. Other complications occurred as follows: bronchitis, six; parotitis, three; intestinal haemorrhage with recovery, three; pneumonia, two; paralysis of the urinary bladder, two; typhoid meningism, one; and severe stomatitis, one." (12)

Stuart and Pullen recently reviewed their experience in New Orleans with 360 patients who contracted typhoid fever during the interval from 1939 through 1944 (13). Almost a third of these persons had received sulfonamide derivatives for various reasons, most frequently to control secondary infections, while the remainder received other therapeutic agents or only supportive treatment. The unequivocal failure of all therapeutic agents to modify the fundamental course of the disease allows the consolidation of both treated and untreated groups in calculating the incidence of various complications. On this basis, 46 of the 360 patients died, yielding a mortality rate of 12.8 per cent. Intestinal hemorrhages were observed in 21 per cent ; intestinal perforations, in 1.9 per cent ; relapses, in 12.5 per cent; and the carrier state, in 1.9 per cent.

McCrae, in 1907 (14), collected statistics from a large group of typhoid patients who were observed in European and American hospitals. In his series intestinal hemorrhage occurred in 7 per cent of 23,271 patients ; perforation, in 3.1 per cent of 34,916 ; and relapses, in 8.8 per cent of 28,057 .

\section{DISCUSSION AND SUMMARY}

Twenty-four patients with typhoid fever were treated with chloramphenicol. There were no fatalities. In one group, consisting of 22 patients, therapy was begun within the first three weeks of illness, average 11.9 days, while the two members of the other group first received the drug during a relapse which occurred in the fifth or seventh week. The average duration of fever after the initial dose of drug was 3.5 days in the first group and 2.5 days in the second. The majority of the patients in the first group recovered without complications, but two had intestinal hemorrhage, one had intestinal perforation, and four had clinical relapses. The group of 22 patients received on the average a total of $23.4 \mathrm{gm}$. of drug given over 9.2 days. It should be noted that the relapses developed in individuals who received less than the average course of therapy, and that they were readily controlled when drug was again administered.

It is evident from the study of this first series of patients that chloramphenicol is a valuable therapeutic agent in the treatment of typhoid fever. It must be realized, however, that the patients become afebrile before the intestinal lesions are healed and, as a result, hemorrhage and perforation may occur after defervescence. The present observations indicate that a relatively high incidence of relapses may be expected if chloramphenicol therapy is restricted to a period of less than eight days.

None of the patients who received chloramphenicol therapy became chronic typhoid carriers. In nine of the 22 persons treated during their primary illness, $S$. typhosa were never recovered from the stools and in none of the 24 persons in this series were typhoid organisms ever found in the urine. Five individuals had $S$. typhosa in their feces after drug therapy was discontinued. Four of these developed clinical relapses. Eventually the stools of all five became free of $S$. typhosa, the last positive culture being obtained on the 50th day after onset.

Large doses of chloramphenicol given over a period of two weeks to four typhoid carriers failed to eradicate permanently the carrier state. During the treatment, when the level of chlorampheni- 
col in the bile was about half that in the blood, no $S$. typhosa were grown from specimens of biliary fluid. Typhoid organisms were again cultivated from the bile shortly after the drug was discontinued. It is worth mentioning that concentrations of chloramphenicol as high as 1,000 gamma/cc. have no detectable in vitro bactericidal effect on $S$. typhosa (our unpublished data). Furthermore, in typhoid fever, as in scrub typhus $(6,9)$, chloramphenicol therapy apparently only suppresses the growth of the organism; ultimate recovery seems dependent on the development of immunity in the host. Therefore, although not anticipated, the discouraging results in typhoid carriers should probably have been expected.

The results in four patients with typhoid fever treated with aureomycin and in five given polymyxin indicated that little value was obtained from these antibiotics. Indeed, the course was essentially unaltered from that of the nine patients with typhoid fever who received only supportive therapy in the present investigation or the large numbers of patients reported in earlier studies by others.

\section{ACKNOWLEDGMENTS}

The authors wish to thank Dr. R. Lewthwaite, Director of the Institute for Medical Research, Kuala Lumpur, and members of the staff of the Institute, particularly Drs. R. Green and D. S. Mankikar, for their help and advice. In addition, the authors wish to express their appreciation of the assistance afforded by the members of the Pediatric and Medical Staffs of the University Hospital, Baltimore, Md. A number of the patients were made available for study by Major C. J. Williams and Lt. H. M. Giles of the Military General Hospital, Kuala Lumpur; Drs. F. L. Choon and C. N. Seong of the General Hospital, Kuala Lumpur; Dr. F. B. Bang, Johns Hopkins Hospital, Baltimore, Md.; Dr. R. V. Campbell, Hagerstown, Md.; and Dr. F. A. Camalier, Leonardtown, Md. Miss Ann Meredith, Research Laboratory, Department of Medicine, University Hospital, Baltimore, contributed technical assistance.

\section{BIBLIOGRAPHY}

1. Woodward, T. E., Smadel, J. E., Ley, H. L., Jr., Green, R., and Mankikar, D. S., Preliminary report on the beneficial effect of Chloromycetin in the treatment of typhoid fever. Ann. Int. Med., 1948, 29, 131.
2. Joslyn, D. A., and Galbraith, M., A turbidimetric method for the assay of antibiotics. J. Bact., 1947, 54, 26.

3. Smith, R. M., Joslyn, D. A., Gruhzit, O. M., McLean, I. W., Jr., Penner, M. A., and Ehrlich, J., Chloromycetin: biological studies. J. Bact. 1948, 55, 425.

4. Collins, H. S., Paine, T. F., Wells, E. B., and Finland, M., Aureomycin-a new antibiotic: evaluation of its effects in typhoid fever, severe salmonella infections and in a case of colon bacillus bacteremia. Ann. Int. Med., 1948, 29, 1077.

5. Smadel, J. E., Woodward, T. E., and Bailey, C. A., Relation of relapses in typhoid to duration of chloramphenicol therapy. J. A. M. A., 1949, 141, 129.

6. Smadel, J. E., Traub, R., Ley, H. L., Jr., Philip, C. B., Woodward, T. E., and Lewthwaite, R., Chloramphenicol (Chloromycetin) in the chemoprophylaxis of scrub typhus (tsutsugamushi disease). II. Results with volunteers exposed in hyperendemic areas of scrub typhus. Am. J. Hyg., 1949, 50, 75.

7. Smadel, J. E., León, A. P., Ley, H. L., Jr., and Varela, G., Chloromycetin in the treatment of patients with typhus fever. Proc. Soc. Exper. Biol. \& Med., 1948, 68, 12.

8. Smadel, J. E., Woodward, T. E., Ley, H. L., Jr., Philip, C. B., Traub, R., Lewthwaite, R., and Savoor, S. R., Chloromycetin in the treatment of scrub typhus. Science, 1948, 108, 160.

9. Smadel, J. E., Woodward, T. E., Ley, H. L., Jr., and Lewthwaite, R., Chloramphenicol (Chloromycetin) in the treatment of tsutsugamushi disease (scrub typhus). J. Clin. Invest., 1949, 28, 1196.

10. Schoenbach, E. B., Bryer, M. S., and Long, P. H., The clinical use of polymyxin. Ann. N. Y. Acad. Sci., 1949, 51, 987.

11. Bliss, E. A., Chandler, C. A., and Schoenbach, E. B., In vitro studies of polymyxin. Ann. N. Y. Acad. Sci., 1949, 51, 944.

12. Green, R., An epidemic of typhoid fever, in: Annual Report of the Institute for Medical Research for 1939, published by Federated Malay States Government Press, 1940, p. 25.

13. Stuart, B. M., and Pullen, R. L., Typhoid; clinical analysis of 360 cases. Arch. Int. Med., 1946, 78, 629.

14. McCrae, T., The symptoms of typhoid fever, in: Osler, W., and McCrae, T., Modern Medicine, Vol. II, Infectious Diseases. Lea Brothers \& Co., Philadelphia, 1907, Chapter IV, p. 104. 